\title{
COMPARISON PRINCIPLE FOR GREEN FUNCTION OF A FOURTH ORDER BOUNDARY VALUE PROBLEM ON A GRAPH
}

\author{
R.CH. KULAEV
}

\begin{abstract}
In the work we develop the disconjugacy theory for fourth order equations on a geometric graph arising in modelling of rod junctions. The disconjugacy of an equation is defined in terms of the properties of a special fundamental system of solutions to the homogeneous equation. We describe the relation between disconjugacy property and the positivity of Green function to some classes of boundary value problems for fourth order equation on a graph.
\end{abstract}

Keywords: graph, differential equation on a graph, disconjugacy, Green function.

Mathematics Subject Classification: 34C10

\section{INTRODUCTION}

The issue on disconjugacy of a differential equation is among the central ones in the qualitative theory of ordinary differential equations [1]-[4]. We recall that in the classical theory an equation of $n$th order is called disconjugate a segment $I \subset \mathbb{R}$ if each its nontrivial solution has at most $n-1$ zeroes counting multiplicities [1], 2]. This property becomes important in studying oscillatory properties of the spectrum of boundary value problems and first of all in studying the positivity of the Green function for various classes of boundary value problems. For instance, for a second order equation in a segment $[a, b] \subset \mathbb{R}$ the disconjugacy is equivalent, on the one hand, to the existence of a positive on the semi-interval $[a, b)$ solution to the homogeneous equation, and on the other hand, to the positivity of the Green function for the Dirichlet problem.

The known nowadays results related with disconjugacy of equations on graph concern first of all second order equations and are exposed in details in monographs [5], 6]. The disconjugacy property of a second order equation on a graph is based on the notion of $S$-zone being an analogue of the interval between neighbouring zeroes of a continuous on a segment function. Given a continuous on a graph function, by $S$-zone we mean any subgraph, on which function has no zeroes and vanishes on its boundary. A second order equation is called disconjugate if each its nontrivial solution has no $S$-zones in the graph. Replacement of "number of zeroes" by "number of $S$-zones" in the definition of disconjugacy for second order equation allows us to obtain an exact analogue of disconjugacy theory for an equation in a segment for second order equations on a graph; namely, analogues of Sturm comparison theorems and Vallée-Poussin disconjugacy criterion. At that, as in the case of an equation in a segment, disconjugacy conditions for a second order equation on a graph turns out to be equivalent on one hand,

R.Ch. Kulaev, Comparison principle for Green function of a fourth order boundary value PROBLEM ON A GRAPH.

(C)Kulaev R.CH. 2015.

Submitted April 10, 2015. 
to the positivity of solutions to special Dirichlet problems, and on the other hand, to the positivity of Green function for Dirichlet problem. At the same time, the Green function for other boundary conditions can be non-positive.

In the present work we study disconjugacy property for fourth order equations on a graph arising in modeling elastic rod constructions. In work [7], a criterion of positivity of the Green function for some boundary value problems for such equations was given. In accordance with [7], the positivity of Green function is equivalent to a positive solvability of specific boundary value problems. This result and the above arguments suggest to choose the positivity property of some fundamental system of solutions to equations on a graph as a starting point in studying disconjugacy property for equations on a graph. Moreover, the fundamental system of solutions characterizes globally a differential equation. Under such approach, specific features of fourth order equations become apparent. While for a second order equation the space of its solutions is parametrized by boundary values of functions, for a fourth order equation a solution is determined uniquely by pairs of values of the solution and its first (or second) derivative at the boundary vertices of the graph. Since at each boundary vertex we have two parameters, for a fourth order equation two types of disconjugacy arise, a weak and a strong one. For the classical case, when there are no internal vertices on a graph, this notions are same. If the set of internal vertices of a graph is non-empty, these notions are different in the general situation. In this work we introduce the notion of a weakly disconjugate equation and study the implications of this property. As we shall show, a weak disconjugacy ensures the positivity of the Green function for a class of Dirichlet problems. Besides it, the property of weak disconjugacy allows us to obtain the comparison principle for the Green functions of Dirichlet boundary value problems.

\section{Main DEFinitions And notations}

In the present work by a geometric graph $\Gamma$ we mean a connected set having a net structure and embedded into $\mathbb{R}^{2}$. An edge of a graph is an interval in $\mathbb{R}^{2}$, while its vertex is a point being an end-point for one or several edges. All the edges are indexed and denoted by $\gamma_{i}$, and the vertices are denoted by $a, b, c$ or $a_{j}, b_{j}, c_{j}$. At that we assume that the indexation of the vertices is independent of that for the edges.

We denote by $J(\Gamma)$ the set of graph vertices being the end-points for two or more edges. Such vertices are called internal vertices. The vertices not belonging to $J(\Gamma)$ will be called boundary vertices and the set of them is denoted by $\partial \Gamma$. We assume that graph $\Gamma$ is the union of the set of all its edges $\gamma_{i}$ and the set of its internal vertices $J(\Gamma)$. At that, the boundary vertices do not belong to the graph. If a vertex $a$ is an end-point of an edge $\gamma_{i}$, we shall say that edge $\gamma_{i}$ is joined to vertex $a$. Sometimes it will be convenient to denote an edge joined to a boundary vertex $a \in \partial \Gamma$ by $\gamma_{a}$. The set of indices of all edges joined to a vertex $a$ is denoted by $I(a)$. The set obtained by removing all the vertices from the graph is denoted by $\Gamma^{\circ}$. A subgraph of a graph $\Gamma$ is any connected subset of $\Gamma$.

As a function on a graph we mean a mapping $u: \stackrel{\circ}{\Gamma} \rightarrow \mathbb{R}$. By $u_{i}(x)$ we denote the restriction of function $u(x)$ on an edge $\gamma_{i}$. By $C[\Gamma]$ we denote the space of functions uniformly continuous on each edge of graph $\Gamma$. For such functions, in each edge $a$ of the graph as $i \in I(a)$ there exists a limit $\lim _{\gamma_{i} \ni x \rightarrow a} u_{i}(x)$, which we denote by $u_{i}(a)$. At that, for an internal vertex $a$, quantities $u_{k}(a)$ an $u_{i}(a)$ are not necessarily same as $k \neq i(k, i \in I(a))$. In space $C[\Gamma]$ we choose the subspace of functions satisfying $u_{k}(a)=u_{i}(a)$ for each $a \in J(\Gamma)$ and each $k, i \in I(a)$. The set of such functions is denoted by $C(\Gamma)$ and they are called continuous on the graph. Such definition is quite natural since we can redefine them by the continuity by letting $u(a)=u_{i}(a)$, $i \in I(a)$. 
Let $a$ be a boundary vertex of a graph and $\gamma_{a}$ be a joined edge. Sometimes we shall employ the phrase "in the vicinity of vertex $a \in \partial \Gamma$ " instead of the phrase "in some semi-neighbourhood of vertex a contained in $\gamma_{a}$ ".

Let us define the notion of the derivative of a function defined on a graph. In order to do it, we introduce a function $\mu(x) \in C[\Gamma]$ mapping each edge $\gamma_{i} \subset \Gamma$ in one-to-one manner onto some interval $\left(0, l_{i}\right) \subset \mathbb{R}$ for $l_{i}>0$. Function $\mu(x)$ is called a metric one, while quantity $l_{i}$ is called the length of edge $\gamma_{i}$. For the restriction of function $\mu(x)$ on edge $\gamma_{i}$ there exists an inverse mapping $x(\mu)$ of interval $\left(0, l_{i}\right)$ on edge $\gamma_{i}$. The metric function defines an orientation on each edge of graph. Funtion $u(x) \in C[\Gamma]$ is called differentiable on graph $\Gamma$ if for each edge $\gamma_{i} \subset \Gamma$ its restriction $u_{i}(x)$ is differentiable w.r.t. $\mu_{i}(x)$. At that we let

$$
u_{i}^{\prime}\left(x_{0}\right)=\left.\frac{d u_{i}(x)}{d \mu_{i}(x)}\right|_{x=x_{0}}=\lim _{\gamma_{i} \ni x \rightarrow x_{0}} \frac{\triangle u_{i}(x)}{\triangle \mu_{i}(x)}, \quad x_{0} \in \bar{\gamma}_{i} .
$$

In the same way we define higher derivatives. We note that the derivatives of odd order depend on the orientation of graph edges, i.e., they are defined up to the sign, while for the derivatives of even orders the orientation plays no role. We denote by $C^{n}[\Gamma]$ the space of functions in $C[\Gamma]$, whose derivatives of orders up to $n$ exist and belong to space $C[\Gamma]$. Space $C^{n}[\Gamma]$ has the natural norm $\|u\|=\sum_{j=0}^{n} \sup _{x \in \stackrel{\Gamma}{\Gamma}}\left|u^{(j)}(x)\right|$.

Following [5], by a differential equation on graph we mean the set of usual differential equations on the graph edges and the set of matching conditions in internal vertices, which can be written generally as

$$
L u=f(x), \quad x \in \Gamma .
$$

While studying differential equations on graphs, an important role is played by the issue on for what connection conditions at the graph vertices one should expect such key properties of the equation like the location of extremum points (maximum principle), positivity of the Green function for the corresponding boundary value problem, properties of the spectrum, etc. It is clear that such properties are unlikely to be possible for all conditions. This is why the basis for selecting classes of conditions at the graph vertices together with those an equation on the graph inherits key properties of an equation on a segment should be the mathematical models of real physical phenomena. Exactly this argument is taken as a premise in selecting classes of 4th order equations on a graph, for which we study analogues of properties of scalar equations on a segment.

In the present work we consider an equation generated by the set of differential equations on graph edges

$$
\left(p_{i}(x) u_{i}^{\prime \prime}\right)^{\prime \prime}-\left(q_{i}(x) u_{i}^{\prime}\right)^{\prime}=f_{i}(x), \quad x \in \gamma_{i} \subset \stackrel{\circ}{\Gamma},
$$

with the coefficients described by functions $p(x) \in C^{2}[\Gamma], \inf _{x \in \Gamma} p(x)>0, q(x) \in C^{1}[\Gamma], q(x) \geqslant 0$ on $\Gamma, f(x) \in C[\Gamma]$, completed by the identities

$$
\begin{gathered}
u_{i}(a)=u_{k}(a), \quad u_{i}^{\prime}(a)=\alpha_{k i}(a) u_{k}^{\prime}(a)+\alpha_{j i}(a) u_{j}^{\prime}(a), \\
\sum_{i \in I(a)} p_{i}(a) \alpha_{k i}(a) u_{i}^{\prime \prime}(a)=0, \quad \sum_{i \in I(a)} p_{i}(a) \alpha_{j i}(a) u_{i}^{\prime \prime}(a)=0,
\end{gathered}
$$

in each internal vertex $a \in J(\Gamma)$ and by the conditions with third derivatives

$$
\sum_{i \in I(a)} D^{3} u_{i}(a)+\delta(a) u(a)=f(a), \quad a \in J(\Gamma)
$$


At that, we assume that in conditions (3), (4) all the derivatives are calculated in the direction from vertex $a \in J(\Gamma) ; k, j$ are fixed (basis) indices in $I(a), i \in I(a) ; \alpha_{k i}(a), \alpha_{j i}(a)$ and $\delta(a)$, $f(a)$ are given numbers, at that $\alpha_{k k}(a)=\alpha_{j j}(a)=1$ and $\alpha_{k j}(a)=\alpha_{j k}(a)=0, \delta(a) \geqslant 0$, while in (4) by $D^{3} u$ we denote the third quasi-derivative $\left(p(x) u^{\prime \prime}\right)^{\prime}-q(x) u^{\prime}$. Moreover, if just two edges $\gamma_{i}$ and $\gamma_{k}$ are joined to an internal vertex $a$, we assume that all the quantities and relation corresponding to index $j$ are absent in the system of conditions (3), (4). In this case conditions (3), (4) become

$$
\begin{gathered}
u_{i}(a)=u_{k}(a), \quad u_{i}^{\prime}(a)=\alpha_{k i}(a) u_{k}^{\prime}(a), \\
\alpha_{k i}(a) p_{i}(a) u_{i}^{\prime \prime}(a)+p_{k}(a)(a) u_{k}^{\prime \prime}(a)(a)=0, \quad D^{3} u_{i}(a)+D^{3} u_{k}(a)+\delta(a) u(a)=f(a) .
\end{gathered}
$$

Thus, left hand side $L u$ of equation (1) are the left hand sides of equations (2) on edges together with identities (3) and the left hand sides of conditions (4) on $J(\Gamma)$, while the right hand side is

$$
F(x)= \begin{cases}f(x), & \text { if } x \in \stackrel{\circ}{\Gamma} \\ f(a), & \text { if } a \in J(\Gamma) .\end{cases}
$$

A solution to differential equation (1) is any function $u(x) \in C^{4}[\Gamma]$ satisfying associated equation (2) on each edge of graph and conditions (3), (4) at each internal vertex.

While studying the properties of solutions to equation (1), we shall assume that the following conditions hold true:

- $p(x) \in C^{2}[\Gamma], \inf _{x \in \Gamma} p(x)>0, q(x) \in C^{1}[\Gamma], q(x) \geqslant 0$ on $\Gamma, f(x) \in C[\Gamma]$

- graph $\Gamma$ is a tree;

- for each vertex $a \in J(\Gamma)$ and each index $i \in I(a)$ at least one of constants $\alpha_{j i}(a), \alpha_{k i}(a)$ is non-zero;

- for each vertex $a \in J(\Gamma)$ there exist basis indices $j, k \in I(a)$ such that for some index $i \in I(a) \backslash\{j, k\}$ the inequalities $\alpha_{j i}(a) \leqslant 0, \alpha_{k i}(a) \leqslant 0$ hold true simultaneously and at least one of the inequalities is strict.

Equation (1) has a natural physical interpretation [9]. It appears while modeling small deformations (not taking into consideration rotation deformations) of a planar mechanical system consisting of thin straight rods and having a rod structure. The junctions of the system are formed by rigid junction of three and more different rods. At that it is assumed that at some points (not necessarily junction or boundary points) the system is elastic backed. This is why the first series of assumption comes from the physical meaning of the problem. Two latter assumptions are to be explained.

We impose continuity conditions of the form $u_{i}(a)=u_{k}(a)$ for each internal vertex by going over all possible indices $i, k \in I(a)$. However, just $|I(a)|-1$ conditions among them are meaningful, where $|I(a)|$ is the number of graph edges joined to a vertex $a$. For instance, such conditions are those with a fixed index $k \in I(a)$ and all $i \in I(a) \backslash k$. Similar assertions can be made for the conditions for the first derivatives in (4), which physically describes the coplanarity of all triples of tangent vectors $u_{i}^{\prime}(a), i \in I(a)$ : if $\theta_{i s}$ is the angle between the references lines of $i$-th and $s$-th rods joined to a vertex $a \in J(\Gamma)$, then $\alpha_{j i}(a)=-\frac{\sin \theta_{k i}}{\sin \theta_{k j}}, \alpha_{k i}(a)=-\frac{\sin \theta_{i j}}{\sin \theta_{k j}}$, at that, fixed indices $k$ and $j$ are chosen so that $0<\theta_{k j}<\pi$. This is why the assumption that for each vertex $a \in J(\Gamma)$ and each index $i \in I(a)$ at least one of constants $\alpha_{j i}(a), \alpha_{k i}(a)$ is non-zero means that the reference lines of different rods do not overlap. This assumption implies that the conditions for the first derivatives can be replaced by their linear combinations of the same form such that any prescribed index $i \in I(a)$ can be made one of the basis ones. Concerning the second basis index, it can not be always arbitrary. Such situation arises in the case, when in the given conditions for the first derivatives in (4) one of constants $\alpha_{k i}(a), \alpha_{j i}(a), i \neq j, k$, 
vanishes: for instance, if for some index $i \in I(a) \backslash\{j, k\}$ the identity $\alpha_{j i}(a)=0$ holds true, then indices $i$ and $k$ can not be simultaneously basis ones. It follows that among all possible options of smoothness conditions, just $|I(a)|-2$ of them are meaningfull, for instance, when two possible basis indices $j, k \in I(a)$ are fixed and $i \in I(a) \backslash\{j, k\}$.

In view of the above arguments and the results of works [8], [10], the latter of our assumptions guarantees the non-degeneracy of the boundary value problem for equation (1) on graph $\Gamma$ (or on any its subgraph $\left.\Gamma_{0} \subset \Gamma\right)$ subject to the boundary conditions

$$
\begin{gathered}
u(a)=0, \quad \vartheta(a) u^{\prime}(a)-\beta(a) u^{\prime \prime}(a)=0, \quad a \in \partial \Gamma\left(a \in \partial \Gamma_{0}\right), \\
\vartheta(a), \beta(a) \geqslant 0, \quad \vartheta(a)+\beta(a)>0 .
\end{gathered}
$$

In the boundary conditions we always assume that each boundary edge of graph $\Gamma$ (of subgraph $\left.\Gamma_{0}\right)$ is oriented from the boundary vertex, which it is joined to.

\section{Fundamental System of SOlutions to EQUation (1)}

The unique solvability of boundary value problem (1), (5) provides the possibility to parametrize the space of solutions to equation (1) on a graph by the pairs of values of a function and its first (second) derivative at boundary vertices of the graph.

Lemma 1. The correspondence between the solutions to homogeneous differential equation (1) and the ordered pairs of the values of solutions and their first (or second) derivative at the boundary is one-to-one.

Proof. By the assumptions for the coefficients of differential equation (1) and boundary conditions (5) as well as by the results of works [8], [10], homogeneous boundary value problem (1), (5) has the trivial solution only. Therefore, inhomogeneous boundary value problem (1), (5) is uniquely solvable. It means that the mapping

$$
u \mapsto\left\{\left(u(a), u^{\left(\omega_{a}\right)}(a)\right)\right\}_{a \in \partial \Gamma},
$$

where $\omega_{a}=1$ or $\omega_{a}=2$, is invertible and sujective, and therefore, it is a one-to-one mapping. The proof is complete.

Corollary 1. Let $|\partial \Gamma|=m$. Then the dimension of the space of solutions to homogeneous differential equation (1) is equal to $2 \mathrm{~m}$.

Corollary 2. Let $|\partial \Gamma|=m$. Then the set of all solutions to homogeneous equation (1) satisfying $n$ conditions of the complete set of boundary conditions (5) form a subspace of dimension $2 m-n$.

\section{WEAK DISCONJUGACY OF EQUATION (1)}

First we study qualitative properties of solutions to homogeneous equation (1) vanishing at the whole boundary of a graph. As the results of work [7] show, the properties of such functions determine the sign of the Green function for the Dirichlet problem for equation (1).

For each vertex $a \in \partial \Gamma$ we introduce the Dirichlet boundary value problem

$$
\begin{gathered}
L u=0, \quad x \in \Gamma, \\
u(a)=0, \quad \vartheta(a) u^{\prime}(a)-\beta(a) u^{\prime \prime}(a)=1, \\
u(b)=0, \quad \vartheta(b) u^{\prime}(b)-\beta(b) u^{\prime \prime}(b)=0, \quad b \in \partial \Gamma \backslash a .
\end{gathered}
$$

It follows from Lemma 1 and Corollaries 1 , 2 that for each vertex $a \in \partial \Gamma$ boundary value problem (6) is uniquely solvable. The results of work [7] imply that the Green function of boundary value problem (1), (5) is strictly positive on $\Gamma \times \Gamma$ if and only if for each boundary 
vertex $a \in \partial \Gamma$ the solution to boundary value problem (6) is positive on the whole graph $\Gamma$ (see [7]). It is clear that the solution to boundary value problem (6) depend on the values of functions $\vartheta(\cdot), \beta(\cdot): \partial \Gamma \rightarrow \mathbb{R}_{+}$determining the coefficients in boundary conditions (5). It was shown in work [11] that if the boundary of graph $\Gamma$ consists of two vertices only, i.e., if graph $\Gamma$ consists of two tandem edges forming a chain, then the sign-definiteness of solutions to boundary value problems (6), $a \in \partial \Gamma$ has no relation with the values of coefficients $\vartheta(\cdot)$ and $\beta(\cdot)$, but depends only of the coefficients of differential equation (1). If the graph is not homeomorphic to an interval, the situation is completely different. Under varying the coefficients of boundary conditions, the solution to boundary value problem (6) (together with the Green function) can loose the positivity property, or, vice versa, to gain it, as the following example shows.

Example. Let us consider a planar graph $\Gamma \subset \mathbb{R}^{2}$ consisting of three edges $\gamma_{i}=\left(a_{i}, b\right)$, $i=1,2,3$, having a joint end-point $b$, which is an internal vertex of the graph. Supposing that edges $\gamma_{i}$ are oriented from boundary vertices $a_{i}$, we consider the homogeneous equation

$$
\begin{gathered}
u^{I V}=0, \quad x \in \gamma_{i}, \\
u_{1}(b)=u_{2}(b)=u_{3}(b), \quad u_{1}^{\prime}(b)=-u_{2}^{\prime}(b)-u_{3}^{\prime}(b), \\
-u_{1}^{\prime \prime}(b)+u_{2}^{\prime \prime}(b)=0, \quad-u_{1}^{\prime \prime}(b)+u_{3}^{\prime \prime}(b)=0, \quad \sum_{i=1}^{3} u_{i}^{\prime \prime \prime}(b)=0,
\end{gathered}
$$

on $\Gamma$. For the lengths $l_{i}$ of graph edges we let $l_{1}=l_{2}=1, l_{3}=\frac{1}{5}$. We consider the boundary value problem for equation (7) subject to the hinged-clamped boundary condition at each boundary vertex $u\left(a_{i}\right)=u^{\prime \prime}\left(a_{i}\right)=0, i=1,2,3$. It was shown in [14] that the corresponding Green function is positive, while it was shown in [7] that the positivity of the Green function for problem (1), (5) is equivalent to the positivity on $\Gamma$ of all solutions to boundary value problems (6). Therefore, if $\vartheta(\cdot) \equiv 0$ in boundary conditions (6), then the solutions to problems (6) for equation (7) are positive on $\Gamma$.

Let us consider solution $y_{a_{1}}(x)$ to boundary value problem (6) for equation (7) with condition of rigid fixing (case $\beta(\cdot) \equiv 0)$, which corresponds to vertex $a_{1} \in \partial \Gamma$. Employing metric function $\mu=\mu(x)$, we have

$$
y_{a_{1}}(x)= \begin{cases}Y_{11} \frac{\mu^{3}(x)}{6}+Y_{12} \mu^{2}(x)+\mu(x), & x \in \gamma_{1} ; \\ Y_{j 1} \frac{\mu^{3}(x)}{6}+Y_{j 2} \frac{\mu^{2}(x)}{2}, & x \in \gamma_{j}, \quad j=2,3 .\end{cases}
$$

Straightforward calculations show that

$$
\begin{aligned}
Y_{11} & \approx 3.681, Y_{12} \approx-3.184 \\
Y_{21} \approx 0.681, Y_{22} & \approx-0.184 \quad Y_{31} \approx-4.362, Y_{32} \approx 1.37
\end{aligned}
$$

It is easy to see that

$$
y_{a_{1}}^{\prime \prime}\left(a_{2}\right)=\left.\frac{d^{2}}{d \mu(x)^{2}}\left(Y_{21} \frac{\mu^{3}(x)}{6}+Y_{22} \frac{\mu^{2}(x)}{2}\right)\right|_{x=a_{2}}=Y_{22}<0 .
$$

Since $y_{a_{1}}\left(a_{2}\right)=0$, we conclude that function $y_{a_{1}}(x)$ is negative in the vicinity of boundary vertex $a_{2}$.

We consider two pairs of nonnegative functions $\beta(\cdot), \widetilde{\beta}(\cdot)$ and $\vartheta(\cdot), \widetilde{\vartheta}(\cdot)$ defined on $\partial \Gamma$ and satisfying the conditions

$$
\beta(a)+\vartheta(a)>0, \quad \widetilde{\beta}(a)+\widetilde{\vartheta}(a)>0, \quad a \in \partial \Gamma .
$$


Each pair $\beta(\cdot), \vartheta(\cdot)$ and $\widetilde{\beta}(\cdot), \widetilde{\vartheta}(\cdot)$ defines a set of boundary value problems $(6), a \in \partial \Gamma$, whose solutions are denoted by $z_{a}(x)$ and $\widetilde{z}_{a}(x)$.

Lemma 2. Assume that the identity $\frac{\beta(a)}{\vartheta(a)} \geqslant \frac{\widetilde{\beta}(a)}{\widetilde{\vartheta}(a)}$ holds true on $\partial \Gamma$ (in the case $\vartheta(a)=0$ we let $\frac{\beta(a)}{\vartheta(a)}=\infty$ and the same is done as $\left.\widetilde{\vartheta}(a)=0\right)$. If all functions $\widetilde{z}_{a}(x), a \in \partial \Gamma$, are positive on graph $\Gamma$, then all functions $z_{a}(x)$ are positive on $\Gamma$.

Proof. If $\frac{\beta(\cdot)}{\vartheta(\cdot)} \equiv \frac{\widetilde{\beta}(\cdot)}{\widetilde{\vartheta}(\cdot)}$ on $\partial \Gamma$, by the non-degeneracy of problems (6) we have $z_{a}(x) \equiv \widetilde{z}_{a}(x)>0$ for each $a \in \partial \Gamma$. This is why the consider the case $\frac{\beta(\cdot)}{\vartheta(\cdot)} \not \equiv \frac{\widetilde{\beta}(\cdot)}{\widetilde{\vartheta}(\cdot)}$. We prove by induction in the number of boundary vertices of the graph, in which the strict inequality $\frac{\beta(\cdot)}{\vartheta(\cdot)}>\frac{\widetilde{\beta}(\cdot)}{\widetilde{\vartheta}(\cdot)}$ holds true.

Suppose that the inequality $\frac{\beta(\cdot)}{\vartheta(\cdot)}>\frac{\widetilde{\beta}(\cdot)}{\widetilde{\vartheta}(\cdot)}$ holds true just in one vertex $b \in \partial \Gamma$. The nondegeneracy of boundary value problem (6) implies $z_{b}(x) \equiv \lambda \widetilde{z}_{b}(x)$ for some $\lambda>0$. This is why $z_{b}(x)>0$ on $\Gamma$.

Consider functions $z_{a}(x)$ corresponding to other boundary vertices. We fix an arbitrary boundary vertex $a \in \partial \Gamma \backslash b$ and we define the function $r(x)=z_{a}(x)-\widetilde{z}_{a}(x)$. It is obvious that function $r(x)$ satisfies the same boundary conditions on $\partial \Gamma \backslash\{a, b\}$ as functions $z_{a}(x), \widetilde{z}_{a}(x)$, while at vertex $a$ the homogeneous boundary condition

$$
\vartheta(a) r^{\prime}(a)-\beta(a) r^{\prime \prime}(a)=0
$$

is satisfied. Let us consider the properties of function $r(x)$ at vertex $b$. We observe immediately that since $\widetilde{z}_{a}(x)>0$ on graph $\Gamma$, the boundary conditions $\widetilde{z}_{a}(b)=0$ and $\widetilde{\vartheta}(b) \widetilde{z}_{a}^{\prime}(b)-\widetilde{\beta}(b) \widetilde{z}_{a}^{\prime \prime}(b)=0$ imply that $\widetilde{z}_{a}^{\prime}(b) \widetilde{z}_{a}^{\prime \prime}(b) \geqslant 0$, and hence, $\widetilde{z}_{a}^{\prime}(b) \geqslant 0$ and $\widetilde{z}_{a}^{\prime \prime}(b) \geqslant 0$. Since $\frac{\beta(b)}{\vartheta(b)}>\frac{\widetilde{\beta}(b)}{\widetilde{\vartheta}(b)}$, then $\beta(b)>0$ and $\widetilde{\vartheta}(b)>0$. Therefore function $r(x)$ satisfies

$$
\begin{aligned}
\vartheta(b) r^{\prime}(b)-\beta(b) r^{\prime \prime}(b) & =\vartheta(b) z_{a}^{\prime}(b)-\beta(b) z_{a}^{\prime \prime}(b)-\vartheta(b) \widetilde{z}_{a}^{\prime}(b)+\beta(b) \widetilde{z}_{a}^{\prime \prime}(b) \\
& =\beta(b)\left[-\frac{\vartheta(b)}{\beta(b)} \widetilde{z}_{a}^{\prime}(b)+\widetilde{z}_{a}^{\prime \prime}(b)\right] \geqslant \beta(b)\left[-\frac{\widetilde{\vartheta}(b)}{\widetilde{\beta}(b)} \widetilde{z}_{a}^{\prime}(b)+\widetilde{z}_{a}^{\prime \prime}(b)\right]=0 .
\end{aligned}
$$

Thus, function $r(x)$ solves homogeneous equation (1) and satisfies the boundary conditions

$$
r(b)=0, \quad \vartheta(b) r^{\prime}(b)-\beta(b) r^{\prime \prime}(b) \geqslant 0, \quad r(c)=0, \quad \vartheta(c) r^{\prime}(c)-\beta(c) r^{\prime \prime}(c)=0, \quad c \in \partial \Gamma \backslash b .
$$

Therefore, either $r(x) \equiv 0$ on $\Gamma$ or $r(x) \equiv \lambda \widetilde{z}_{b}(x)$ for some $\lambda>0$. Hence, $r(x) \geqslant 0$ on the graph, i.e., $z_{a}(x) \geqslant \widetilde{z}_{a}(x)>0, a \in \partial \Gamma$.

Suppose that the lemma is true for the case, when the inequality $\frac{\beta(\cdot)}{\vartheta(\cdot)}>\frac{\widetilde{\beta}(\cdot)}{\widetilde{\vartheta}(\cdot)}$ holds true in any $n$ boundary vertices of the graph for some $n \leqslant m-1$ and let us show that then it is true for the case of strict inequality in $(n+1)$-th vertex. We choose arbitrary vertex $b \in \partial \Gamma$, at which the strict inequality $\frac{\beta(b)}{\vartheta(b)}>\frac{\widetilde{\beta}(b)}{\widetilde{\vartheta}(b)}$ holds true. We replace the value of function $\beta(\cdot)$ at point $b \in \partial \Gamma$ on $\widetilde{\beta}(b)$, and value $\vartheta(b)$ is replaced by $\widetilde{\vartheta}(b)$. Then we denote by $\widehat{\beta}(\cdot)$ and $\widehat{\vartheta}(\cdot)$ the obtained in this way functions. By $\widehat{z}_{a}(x), a \in \partial \Gamma$, we denote solutions to boundary value problems (6), $a \in \partial \Gamma$, obtained under the described change. By the induction assumption, for all $a \in \partial \Gamma$ the inequalities $\widehat{z}_{a}(x)>0$ hold true on graph $\Gamma$. For an arbitrary vertex $a \in \partial \Gamma$ we introduce the function $r(x)=z_{a}(x)-\widehat{z}_{a}(x)$ and reproducing literally the above arguments, we show that function $r(x)$ solves homogeneous equation (1) and satisfies the boundary conditions

$$
r(b)=0, \quad \vartheta(b) r^{\prime}(b)-\beta(b) r^{\prime \prime}(b) \geqslant 0, \quad r(c)=0, \quad \vartheta(c) r^{\prime}(c)-\beta(c) r^{\prime \prime}(c)=0, \quad c \in \partial \Gamma \backslash b,
$$


on $\partial \Gamma$. It yields the identity $r(x) \equiv \lambda \widetilde{z}_{a}(x)$ for some $\lambda \geqslant 0$. This is why $r(x) \geqslant 0$ on the graph and hence, $z_{a}(x) \geqslant \widehat{z}_{a}(x)>0$. The proof is complete.

Definition 1. Differential equation (1) is called weakly disconjugate on graph $\Gamma$ if for each boundary vertex $a \in \partial \Gamma$ boundary value problem (6) for $\beta(\cdot) \equiv 0$ has a positive on $\Gamma$ solution.

It should be noted that the definition of weak disconjugacy is extended also for the equations generated by ordinary differential equations (2) on graph edges with pin-joint connection conditions

$$
\begin{array}{cl}
u_{i}(a)=u_{k}(a), \quad \vartheta_{i}(a) u_{i}^{\prime}(a)-\beta_{i}(a) u_{i}^{\prime \prime}(a)=0, \quad i, k \in I(a), a \in J(\Gamma), \\
& \vartheta_{i}(\cdot), \beta_{i}(\cdot) \geqslant 0, \quad \vartheta_{i}(\cdot)+\beta_{i}(\cdot)>0,
\end{array}
$$

and conditions (4) on $J(\Gamma)$ (all the derivatives are calculated from vertices $a \in J(\Gamma)$ ). The properties of solutions to this equation, which we call $\left(1^{\prime}\right)$, were studied in works by Yu.V. Pokornyi, A.V. Borovskich, K.P. Lazarev and R.O. Mustafakulov (see [12, [13] and the references therein) and they are presented in details in monogrpah [5, Ch. 8]. Taking into consideration the results of [5], we can state that equation $\left(1^{\prime}\right)$ is always weakly disconjugate. Indeed, if we replace homogeneous equation (1) by homogeneous equation $\left(1^{\prime}\right)$ in boundary value problem (6) and denote by $v_{a}(x)$ the solution of the obtained problem, then, as it follows from the results of [5], [13], function $v_{a}(x)$ is monotonous on each graph edge except boundary edge $\gamma_{a}=(a, b)$, $a \in \partial \Gamma, b \in J(\Gamma)$. Moreover (see [5], [13]), each solution to this equation satisfying the condition $u^{\prime}(\cdot) u^{\prime \prime}(\cdot) \geqslant 0$ at each boundary vertex, attains the maximum and minimum on the boundary of the graph (maximum principle). Since $v_{a}^{\prime}(a)>0$, then either function $v_{a}(x)$ has a point of maximum $\xi \in \gamma_{a}$ such that $v_{a}^{\prime}(\xi)=0$ and $v_{a}(x)>0$ on $(a, \xi] \subset \gamma_{a}$ or $v_{a}^{\prime}(x)>0$ on $\gamma_{a}$, and function $v_{a}(x)$ increases on edge $\gamma_{a}$. In the former case, by the maximum principle, $v_{a}(x)>0$ on $\Gamma \backslash(a, \xi)$, and hence, $v_{a}(x)>0$ on $\Gamma$. In the latter case, function $v_{a}(x)$ is positive on edge $\gamma_{a}$ and at vertex $b \in J(\Gamma)$. By the maximum principle it follows that $v_{a}(x)>0$ on each graph branch $b \in J(\Gamma)$. Therefore, in any case solution $v_{a}(x), a \in \partial \Gamma$, is positive on $\Gamma$, i.e., equation $\left(1^{\prime}\right)$ is weakly disconjugate.

In terms of weak disconjugacy we succeed to formulate a series of useful properties of solutions to fourth order equations as well as a series of properties of the Green function for the Dirichlet boundary value problems for fourth order equation (1).

Corollary 3. Suppose that differential equation (1) is weakly disconjugate on $\Gamma$. Then a solution to boundary value problem (6) is positive on graph $\Gamma$ for all admissible values of coefficients $\vartheta(\cdot), \beta(\cdot)$ in the boundary conditions.

Lemma 3. Suppose that differential equation (1) is weakly disconjugate on $\Gamma$. Then for each solution of equation (1) vanishing on $\partial \Gamma$, the inequality $u^{\prime}(x) \geqslant 0$ on $\partial \Gamma$ implies $u(x) \geqslant 0$ on $\Gamma$. If, in addition, $u^{\prime}(x) \not \equiv 0$ on $\partial \Gamma$, then $u(x)>0$ on graph $\Gamma$.

The proof is almost obvious: it follows from Lemma 1 and Corollary 3 that function $u(x)$ is a linear combination of positive solutions to boundary value problems $(6), a \in \partial \Gamma$, as $\beta(\cdot) \equiv$ 0 , with non-negative coefficients. At that, if $u^{\prime}(a)>0$ for some vertex $a \in \partial \Gamma$, then the corresponding coefficient in the linear combination is positive.

Lemma 2 and Corollary 3 allow us to formulae the comparison theorem for the Green function of Dirichlet boundary value problem (1), (5).

Theorem 1 (comparison test). Suppose that the inequality $\frac{\beta(a)}{\vartheta(a)} \geqslant \frac{\widetilde{\beta}(a)}{\widetilde{\vartheta}(a)}$ holds true on $\partial \Gamma$ (in the case $\vartheta(a)=0$ we let $\frac{\beta(a)}{\vartheta(a)}=\infty$, and the same for $\left.\widetilde{\vartheta}(a)=0\right)$. Then the positivity of 
the Green function $\widetilde{G}(x, s)$ of boundary value problem (1), (5) with coefficients $\widetilde{\beta}(\cdot)$ and $\widetilde{\vartheta}(\cdot)$ under conditions (5) implies the positivity of Green function $G(x, s)$ of problem (1), (5) with coefficients $\beta(\cdot)$ and $\vartheta(\cdot)$.

Proof. It was shown in work [7] that the Green function for boundary value problem (1), (5) is positive on $\Gamma \times \Gamma$ if and only if for each boundary vertex $a \in \partial \Gamma$ solution $v_{a}(x)$ to problem (6) is positive on graph $\Gamma$. Since $\widetilde{G}(x, s)>0$ on $\Gamma \times \Gamma$, for each $a \in \partial \Gamma$ the strict inequalities $\widetilde{v}_{a}(x)>0$ hold true on graph $\Gamma$. And it follows from Lemma 2 and the assumptions of the theorem that for each $a \in \partial \Gamma$ the inequalities $v_{a}(x)>0$ hold true on graph $\Gamma$ that implies the positivity of function $G(x, s)$. The proof of complete.

As corollaries of the comparison theorem we consider its limiting cases.

Corollary 4. If the Green function for the Dirichlet problem for equation (1) subject to rigid fixing condition on the boundary is positive on $\Gamma \times \Gamma$, then the Green function for Dirichlet boundary value problem (1), (5) is positive for all admissible values of coefficients $\vartheta(\cdot), \beta(\cdot)$ in boundary conditions (5).

Corollary 5. If the Green function of the Dirichlet problem for equation (1) subject to hinged-clamped boundary condition is sign-changing on $\Gamma \times \Gamma$, then the Green function for the Dirichlet problem is also sign-changing for all admissible values of coefficients $\vartheta(\cdot), \beta(\cdot)$ in boundary conditions (5).

As Corollary 4 shows, the property of weak disconjugacy of differential equation (1) is equivalent to the positivity of the Green function for Dirichlet problem (1), (5) for all admissible values of coefficients $\vartheta(\cdot), \beta(\cdot)$ in the boundary conditions.

We should mention the connection of the weak disconjugacy of equation (1) with the theory of Gantmacher-Krein oscillating kernels [15], [16]. If a graph $\Gamma$ is a chain of sequentially joined edges (see [11], [17]), the property of weak disconjugacy is necessary and sufficient for oscillation of the Green function for Dirichlet problem (1), (5).

\section{BIBLIOGRAPHY}

1. A.Yu. Levin. Disconjugacy of solutions of the equation $x^{(n)}+p_{1}(t) x^{(n-1)}+\cdot+p_{n}(t) x=0 / /$ Uspekhi Matem. Nauk. 24:2, 43-96 (1969). [Russ. Math. Surv. 24:2, 43-99 (1969).]

2. V.Ya. Derr. Disconjugacy of solutions to differential equations // Vestn. Udmurt. Univ. 1, 46-89 (2009). (in Russian).

3. A. Wintner. On the non-existence of conjugate points // Amer. J. Math. 73:2, 368-380 (1953).

4. A.L. Teptin. On the oscillation of the spectrum of a multipoint boundary value problem // Izv. VUZov. Matem. 4, 44-53 (1999). [Russ. Math. (Izv. VUZ. Matem.) 43:4, 42-52 (1999).]

5. Yu.V. Pokornyi, O.M. Penkin, V.L. Pryadiev, A.V. Borovskikh, K.P. Lazarev, S.A. Shabrov. Differential equations on geometric graphs. Fizmatlit, Moscow (2007). (in Russian.)

6. Yu.V. Pokornyi, Zh.I. Bakhtina,, M.B. Zvereva, S.A. Shabrov. Sturm's oscillation method for spectral problems. Fizmatlit, Moscow (2009). (in Russian.)

7. R.Ch. Kulaev. Necessary and sufficient condition for the positivity of the Green function of a boundary value problem for a fourth-order equation on a graph // Differ. Uravn. 51:3, 302-316 (2015). [Diff. Equat. 51:3, 303-317 (2015).]

8. R.Ch. Kulaev. On the solvability of a boundary value problem for a fourth-order equation on a graph // Differ. Uravn. 50:1, 27-34 (2014). [Diff. Equat. 50:1, 25-32 (2014).]

9. M.G. Zavgorodnii. Variational principles of constructing models of rods // Matem. Modelir. Informats. Tekhnolog. Sistem. 4, 59-62 (2000). (in Russian.) 
10. R.Ch. Kulaev. Criterion for the positiveness of the Green function of a many-point boundary value problem for a fourth-order equation // Differ. Uravn. 51:2, 161-173 (2015). [Differ. Equat. 51:2, 163-176 (2015).]

11. R.Ch. Kulaev. Oscillatory properties of the Green function of discontinuous boundary value problem for equations of the fourth order // Vladikavkaz. Matem. Zhurn. 17:1, 47-59 (2015). (in Russian.)

12. A.V. Borovskikh, R.O. Mustafokulov, K.P. Lazarev, Yu.V. Pokornyi. A class of fourth-order differential equations on a spatial net // Dokl. RAN. 345:6, 730-732 (1995). [Dokl. Math. 52:3, 433-435 (1995).]

13. A.V. Borovskikh, K.P. Lazarev. Fourth-order differential equations on geometric graphs // J. Math. Sci. 119:6, 719-738 (2004).

14. R.Ch. Kulaev. The source function of the chain of rods with elastic supports // Vladikavkaz. Matem. Zhurn. 16:2, 49-61 (2014). (in Russian.)

15. F.R. Gantmacher, M.G. Krein. Oscillation matrices and kernels and small vibrations of mechanical systems. Gostekhizdat, Moscow-Leningrad (1950). [AMS Chelsea Publishing, Providence, RI (2002).]

16. A.Yu. Levin, G.D. Stepanov. One-dimensional boundary value problems for operators not increasing the number of sign changes. II. // Sibir. Matem. Zhurn. 17:4, 813-830 (1976). (in Russian.)

17. R.Ch. Kulaev. Oscillation of the green function of a multipoint boundary value problem for a fourth-order equation // Differ. Uravn. 51:4, 445-458 (2015). [Differ. Equat. 51:4, 449-463 (2015).]

Ruslan Chermenovich Kulaev,

South Mathematical Institute, VSC RAS,

Markus str., 22,

362027, Vladikavkaz, Russia

North-Ossetia State Univeristy named after K.L. Khetagurov,

Vatutin str., 46,

362025, Vladikavkaz, Russian

E-mail: kulaev@smath.ru 